\title{
New Results on Robust Stability and Stabilization of Linear Discrete-Time Stochastic Systems with Convex Polytopic Uncertainties
}

\author{
P. Niamsup ${ }^{1,2}$ and G. Rajchakit ${ }^{3}$ \\ ${ }^{1}$ Department of Mathematics, Faculty of Science, Chiang Mai University, Chiangmai 50200, Thailand \\ ${ }^{2}$ Center of Excellence in Mathematics, CHE, Si Ayutthaya Road, Bangkok 10400, Thailand \\ ${ }^{3}$ Division of Mathematics and Statistics, Faculty of Science, Maejo University, Chiangmai 50290, Thailand \\ Correspondence should be addressed to G. Rajchakit; mrajchakit@yahoo.com
}

Received 17 January 2013; Revised 1 April 2013; Accepted 15 April 2013

Academic Editor: Rung Ching Chen

Copyright ( 2013 P. Niamsup and G. Rajchakit. This is an open access article distributed under the Creative Commons Attribution License, which permits unrestricted use, distribution, and reproduction in any medium, provided the original work is properly cited.

\begin{abstract}
This paper addresses the robust stability for a class of linear discrete-time stochastic systems with convex polytopic uncertainties. The system to be considered is subject to both interval time-varying delays and convex polytopic type uncertainties. Based on the augmented parameter-dependent Lyapunov-Krasovskii functional, new delay-dependent conditions for the robust stability are established in terms of linear matrix inequalities. An application to robust stabilization of linear discrete-time stochastic control systems is given. Numerical examples are included to illustrate the effectiveness of our results.
\end{abstract}

\section{Introduction}

In the past decades, the problem of stability for neutral differential systems, which have delays in both its state and the derivatives of its states, has been widely investigated by many researchers. Such systems are often encountered in engineering, biology, and economics. The existence of time delay is frequently a source of instability or poor performances in the systems. Recently, some stability criteria for neutral system with time delay have been given in [1-8] and the references therein. Some delay-dependent stability criteria for discrete-time systems with time-varying delay are investigated in $[2,6,9-11]$, where the discrete Lyapunov functional method is employed to prove stability conditions in terms of linear matrix inequalities (LMIs). A number of research works for dealing with asymptotic stability problem for discrete systems with interval time-varying delays have been presented in [12-24]. Theoretically, stability analysis of the systems with time-varying delays is more complicated, especially for the case where the system matrices belong to some convex polytope. In this case, the parameter-dependent Lyapunov-Krasovskii functionals are constructed as the convex combination of a set of functions assures the robust stability of the nominal systems, and the stability conditions must be solved upon a grid on the parameter space, which results in testing a finite number of linear matrix inequalities (LMIs) [11, 25, 26]. To the best of the authors' knowledge, the stability for linear discrete-time systems with both timevarying delays and polytopic uncertainties has not been fully investigated. The papers $[27,28]$ propose sufficient conditions for robust stability of discrete and continuous polytopic systems without time delays. More recently, combining the ideas in $[25,26]$, improved conditions for $\mathscr{D}$-stability and D-stabilization of linear polytopic delay-difference equations with constant delays have been proposed in [29]. To the best of our knowledge, the stability and stabilization of linear discrete-time stochastic systems with convex polytopic uncertainties, nondifferentiable time-varying delays has not been fully studied yet (see, e.g., $[1,3-11,13-36]$ and the references therein), which are important in both theories and applications. This motivates our research.

In this paper, we consider polytopic discrete-time stochastic equations with interval time-varying delays. By using the parameter-dependent Lyapunov-Krasovskii functional combined with LMI techniques, we propose new criteria for the robust stability of the stochastic system. The 
delay-dependent stability conditions are formulated in terms of LMIs, being thus solvable by the numeric technology available in the literature to date. The result is applied to robust stabilization of linear discrete-time stochastic control systems. Compared to other results, our result has its own advantages. First, it deals with the delay-difference stochastic system, where the state-space data belong to the convex polytope of uncertainties and the rate of change of the state depends not only on the current state of the systems, but also its state at some times in the past. Second, the time delay is assumed to be a time-varying function belonging to a given interval, which means that the lower and upper bounds for the time-varying delay are available. Third, our approach allows us to apply in robust stabilization of the linear discretetime stochastic system subjected to polytopic uncertainties and external controls. Therefore, our results are more general than the related previous results.

The paper is organized as follows. Section 2 introduces the main notations, definitions, and some lemmas needed for the development of the main results. In Section 3, sufficient conditions are derived for robust stability, stabilization of discrete-time stochastic systems with interval time-varying delays, and polytopic uncertainties. They are followed by some remarks. Illustrative examples are given in Section 4.

\section{Preliminaries}

The following notations will be used throughout this paper. $R^{+}$denotes the set of all real nonnegative numbers; $R^{n}$ denotes the $n$-dimensional space with the scalar product $\langle\cdot, \cdot\rangle$ and the vector norm $\|\cdot\| ; R^{n \times r}$ denotes the space of all real matrices of $(n \times r)$-dimension. $A^{T}$ denotes the transpose of $A$; a matrix $A$ is symmetric if $A=A^{T}$, and a matrix $I$ is the identity matrix of appropriate dimension.

Matrix $A$ is semipositive definite $(A \geq 0)$ if $\langle A x, x\rangle \geq 0$, for all $x \in R^{n}$; $A$ is positive definite $(A>0)$ if $\langle A x, x\rangle>0$ for all $x \neq 0 ; A \geq B$ means that $A-B \geq 0$.

Consider delay-difference stochastic systems with polytopic uncertainties of the form

$$
\begin{array}{r}
x(k+1)=A(\xi) x(k)+D(\xi) x(k-h(k)) \\
+\sigma(x(k), x(k-h(k)), k) \omega(k), \\
k=0,1,2, \ldots, \\
x(k)=v_{k}, \quad k=-h_{2},-h_{2}+1, \ldots, 0,
\end{array}
$$

where $x(k) \in R^{n}$ is the state (Figures 1 and 2), and the system matrices are subjected to uncertainties and belong to the polytope $\Omega$ given by

$$
\Omega=\left\{[A, D](\xi):=\sum_{i=1}^{p} \xi_{i}\left[A_{i}, D_{i}\right], \sum_{i=1}^{p} \xi_{i}=1, \xi_{i} \geq 0\right\},
$$

where $A_{i}, D_{i}, i=1,2, \ldots, p$, are given constant matrices with appropriate dimensions, $\omega(k)$ is a scalar Wiener process (Brownian Motion) on $(\Omega, \mathscr{F}, \mathscr{P})$ with

$$
\begin{gathered}
E[\omega(k)]=0, \quad E\left[\omega^{2}(k)\right]=1, \\
E[\omega(i) \omega(j)]=0 \quad(i \neq j),
\end{gathered}
$$

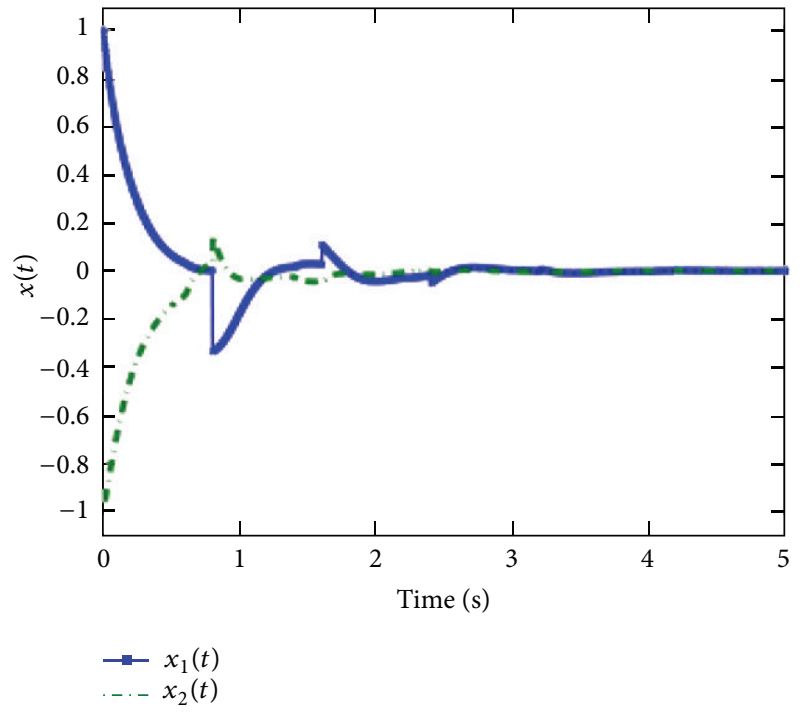

FIGURE 1: The simulation of the solutions $x_{1}(k)$ and $x_{2}(k)$ with the initial condition $\phi(k)=\left[\begin{array}{ll}10 & 5\end{array}\right]^{T}, k \in[0,10]$.

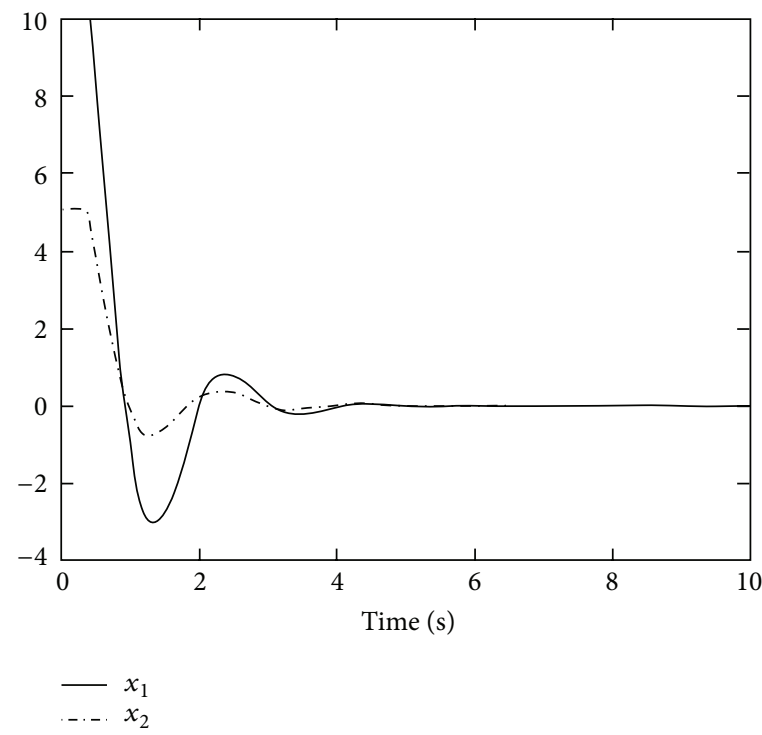

FIGURE 2: The simulation of the solutions $x_{1}(k)$ and $x_{2}(k)$ with the initial condition $\phi(k)=\left[\begin{array}{ll}10 & 5\end{array}\right]^{T}, k \in[0,10]$.

and $\sigma: R^{n} \times R^{n} \times R \rightarrow R^{n}$ is the continuous function and is assumed to satisfy that

$$
\begin{aligned}
& \sigma^{T}(x(k), x(k-h(k)), k) \sigma(x(k), x(k-h(k)), k) \\
& \quad \leq \rho_{1} x^{T}(k) x(k)+\rho_{2} x^{T}(k-h(k)) x(k-h(k)), \\
& x(k), x(k-h(k)) \in R^{n},
\end{aligned}
$$

where $\rho_{1}>0$ and $\rho_{2}>0$ are known constant scalars.

For simplicity, we denote $\sigma(x(k), x(k-h(k)), k)$ by $\sigma$, respectively. 
The time-varying function $h(k)$ satisfies the condition:

$$
0<h_{1} \leq h(k) \leq h_{2}, \quad \forall k=0,1,2, \ldots
$$

Remark 1. It is worth noting that the time delay is a timevarying function belonging to a given interval, which allows the time delay to be a fast time-varying function, and the lower bound is not restricted to being zero as considered in $[2,6,9-11,18-24,30-33]$.

Definition 2. The system (1) is robustly stable in the mean square if there exists a positive definite scalar function $V(k$, $x(k)): R^{n} \times R^{n} \rightarrow R$ such that

$$
\begin{aligned}
E[ & \Delta V(k, x(k))] \\
& =E[V(k+1, x(k+1))-V(k, x(k))]<0,
\end{aligned}
$$

along any trajectory of zero solution of the system (1) for all uncertainties in $\Omega$.
Proposition 3. For real numbers $\xi_{i} \geq 0, i=1,2, \ldots, p$, $\sum_{i=1}^{p} \xi_{i}=1$, the following inequality holds:

$$
(p-1) \sum_{i=1}^{p} \xi_{i}^{2}-2 \sum_{i=1}^{p-1} \sum_{j=i+1}^{p} \xi_{i} \xi_{j} \geq 0
$$

Proof. The proof is followed from completing the square

$$
(p-1) \sum_{i=1}^{p} \xi_{i}^{2}-2 \sum_{i=1}^{p-1} \sum_{j=i+1}^{p} \xi_{i} \xi_{j}=\sum_{i=1}^{p-1} \sum_{j=i+1}^{p}\left(\xi_{i}-\xi_{j}\right)^{2} \geq 0
$$

\section{Main Results}

3.1. Robust Stability. In this section, we present sufficient delay-dependent conditions for the robust stability of system (1). Let us set

$$
\begin{aligned}
& \left\|x_{k}\right\|=\sup _{s \in\left[-h_{2}, 0\right]}\|x(k+s)\|, \\
& \mathscr{M}_{i j}\left(P, Q, S_{1}, S_{2}\right)=\left(\begin{array}{ccc}
\left(h_{2}-h_{1}+1\right) Q_{i}-P_{i}-S_{1 i} A_{j}-A_{j}^{T} S_{1 i}^{T}+2 \rho_{1} I & S_{1 i}-S_{1 i} A_{j} & -S_{1 i} D_{j}-S_{2 i} A_{j} \\
S_{1 i}^{T}-A_{j}^{T} S_{1 i}^{T} & P_{i}+S_{1 i}+S_{1 i}^{T} & S_{2 i}-S_{1 i} D_{j} \\
-D_{j}^{T} S_{1 i}^{T}-A_{j}^{T} S_{2 i}^{T} & S_{2 i}^{T}-D_{j}^{T} S_{1 i}^{T} & -Q_{i}-S_{2 i} D_{j}-D_{j}^{T} S_{2 i}^{T}+2 \rho_{2} I
\end{array}\right), \\
& \mathcal{S}=\left(\begin{array}{ccc}
S & 0 & 0 \\
0 & 0 & 0 \\
0 & 0 & 0
\end{array}\right), \quad P(\xi)=\sum_{i=1}^{p} \xi_{i} P_{i}, \quad Q(\xi)=\sum_{i=1}^{p} \xi_{i} Q_{i}, \quad S_{1}(\xi)=\sum_{i=1}^{p} \xi_{i} S_{1 i}, \quad S_{2}(\xi)=\sum_{i=1}^{p} \xi_{i} S_{2 i} .
\end{aligned}
$$

Theorem 4. The system (1) is robustly stable in the mean square if there exist symmetric matrices $P_{i}>0, Q_{i}>0, i=$ $1,2 \ldots, p$, and constant matrices $S \geq 0, S_{1 i}, S_{2 i}, i=1,2 \ldots, p$, satisfying the following LMIs:

(i) $\mathscr{M}_{i i}\left(P, Q, S_{1}, S_{2}\right)+\mathcal{S}<0, i=1,2, \ldots, p$;

(ii) $\mathscr{M}_{i j}\left(P, Q, S_{1}, S_{2}\right)+\mathscr{M}_{j i}\left(P, Q, S_{1}, S_{2}\right)-(2 /(p-1)) \mathcal{S}<0$, $i=1,2, \ldots, p-1 ; j=i+1, \ldots, p$.

Proof. Consider the following parameter-dependent Lyapunov-Krasovskii functional for system (1):

$$
V(k)=V_{1}(k)+V_{2}(k)+V_{3}(k),
$$

where

$$
\begin{gathered}
V_{1}(k)=x(k) P(\xi) x(k), \\
V_{2}(k)=\sum_{i=k-h(k)}^{k-1} x^{T}(i) Q(\xi) x(i), \\
V_{3}(k)=\sum_{j=-h_{2}+2}^{-h_{1}+1} \sum_{l=k+j+1}^{k-1} x^{T}(l) Q(\xi) x(l) .
\end{gathered}
$$

We can verify that

$$
\lambda_{1}\|x(k)\|^{2} \leq V(k) \leq \lambda_{2}\left\|x_{k}\right\|^{2} .
$$

Let us set $z(k)=\left[x^{T}(k) x^{T}(k+1) x^{T}(k-h(k)) \omega^{T}(k)\right]$, and

$$
G(\xi)=\left(\begin{array}{cccc}
0 & 0 & 0 & 0 \\
0 & P(\xi) & 0 & 0 \\
0 & 0 & 0 & 0 \\
0 & 0 & 0 & 0
\end{array}\right), \quad F(\xi)=\left(\begin{array}{cccc}
P(\xi) & 0 & 0 & 0 \\
I & I & 0 & 0 \\
0 & 0 & I & 0 \\
0 & 0 & 0 & I
\end{array}\right)
$$

Then, with the difference of $V_{1}(k)$ along the solution of the system (1) and taking the mathematical expectation, we obtained

$$
\begin{aligned}
E[ & \left.\Delta V_{1}(k)\right] \\
& =E\left[x^{T}(k+1) P(\xi) x(k+1)-x^{T}(k) P(\xi) x(k)\right] \\
& =E\left[z(k)^{T} G(\xi) z(k)-2 z^{T}(k) F^{T}(\xi)\left(\begin{array}{c}
0.5 x(k) \\
0 \\
0 \\
0
\end{array}\right)\right],
\end{aligned}
$$


because of

$$
\begin{gathered}
z^{T}(k) G(\xi) z(k)=x(k+1)^{T} P(\xi) x(k+1), \\
2 z^{T}(k) F^{T}(\xi)\left(\begin{array}{c}
0.5 x(k) \\
0 \\
0 \\
0
\end{array}\right)=x^{T}(k) P(\xi) x(k) .
\end{gathered}
$$

Using the expression of system (1)

$$
\begin{aligned}
0= & -S_{2}(\xi) x(k+1)+S_{2}(\xi) A(\xi) x(k) \\
& +S_{2}(\xi) D(\xi) x(k-h(k))+S_{2}(\xi) \sigma \omega(k), \\
0= & -\sigma^{T} x(k+1)+\sigma^{T} A(\xi) x(k) \\
& +\sigma^{T} D(\xi) x(k-h(k))+\sigma^{T} \sigma \omega(k),
\end{aligned}
$$

we have

$$
\begin{aligned}
& -2 z^{T}(k) F^{T}(\xi)\left(\begin{array}{c}
0.5 x(k) \\
-S_{1}(\xi) x(k+1)+S_{1}(\xi) A(\xi) x(k)+S_{1}(\xi) D(\xi) x(k-h(k))+S_{1}(\xi) \sigma \omega(k) \\
-S_{2}(\xi) x(k+1)+S_{2}(\xi) A(\xi) x(k)+S_{2}(\xi) D(\xi) x(k-h(k))+S_{2}(\xi) \sigma \omega(k) \\
-\sigma^{T} x(k+1)+\sigma^{T} A(\xi) x(k)+\sigma^{T} D(\xi) x(k-h(k))+\sigma^{T} \sigma \omega(k)
\end{array}\right) z(k) \\
& =-z^{T}(k) F^{T}(\xi)\left(\begin{array}{cccc}
0.5 I & 0 & 0 & \\
S_{1}(\xi) A(\xi) & -S_{1}(\xi) & S_{1}(\xi) D(\xi) & S_{1}(\xi) \sigma \\
S_{2}(\xi) A(\xi) & -S_{2}(\xi) & S_{2}(\xi) D(\xi) & S_{2}(\xi) \sigma \\
\sigma^{T} A(\xi) & -\sigma^{T} & \sigma^{T} D(\xi) & \sigma^{T} \sigma
\end{array}\right) z(k) \\
& -z^{T}(k)\left(\begin{array}{cccc}
0.5 I & 0 & 0 & \\
S_{1}(\xi) A(\xi) & -S_{1}(\xi) & S_{1}(\xi) D(\xi) & S_{1}(\xi) \sigma \\
S_{2}(\xi) A(\xi) & -S_{2}(\xi) & S_{2}(\xi) D(\xi) & S_{2}(\xi) \sigma \\
\sigma^{T} A(\xi) & -\sigma^{T} & \sigma^{T} D(\xi) & \sigma^{T} \sigma
\end{array}\right)^{T} F(\xi) z(k)
\end{aligned}
$$

Therefore, from (14), it follows that

$$
\begin{aligned}
E\left[\Delta V_{1}(k)\right] & \\
=E[ & x^{T}(k)\left[-P(\xi)-S_{1}(\xi) A(\xi)-A(\xi)^{T} S_{1}^{T}(\xi)\right] x(k) \\
& +2 x^{T}(k)\left[S_{1}(\xi)-S_{1}(\xi) A(\xi)\right] x(k+1) \\
& +2 x^{T}(k)\left[-S_{1}(\xi) D(\xi)\right. \\
& \left.\quad-S_{2}(\xi) A(\xi)\right] x(k-h(k)) \\
& +2 x^{T}(k)\left[-S_{1}(\xi) \sigma-\sigma^{T} A(\xi)\right] \omega(k) \\
& +x(k+1)\left[P(\xi)+S_{1}(\xi)+S_{1}^{T}(\xi)\right] \\
& \times x(k+1) \\
& +2 x(k+1)\left[S_{2}(\xi)-S_{1}(\xi) D(\xi)\right] \\
& \times x(k-h(k)) \\
& +2 x(k+1)\left[\sigma^{T}-S_{1}(\xi) \sigma\right] \omega(k) \\
& +x^{T}(k-h(k))\left[-S_{2}(\xi) D(\xi)-D^{T}(\xi) S_{2}^{T}(\xi)\right] \\
& \times x(k-h(k)) \\
& +x^{T}(k-h(k))\left[-S_{2}(\xi) \sigma-\sigma^{T} D(\xi)\right] \omega(k) \\
& \left.+\omega^{T}(k)\left[-2 \sigma^{T} \sigma\right] \omega(k)\right] .
\end{aligned}
$$

By assumption (3), we have

$$
\begin{aligned}
E\left[\Delta V_{1}(k)\right] & \\
=E[ & x^{T}(k)\left[-P(\xi)-S_{1}(\xi) A(\xi)-A(\xi)^{T} S_{1}^{T}(\xi)\right] x(k) \\
& +2 x^{T}(k)\left[S_{1}(\xi)-S_{1}(\xi) A(\xi)\right] x(k+1) \\
& +2 x^{T}(k)\left[-S_{1}(\xi) D(\xi)-S_{2}(\xi) A(\xi)\right] \\
& \times x(k-h(k)) \\
& +x(k+1)\left[P(\xi)+S_{1}(\xi)+S_{1}^{T}(\xi)\right] x(k+1) \\
& +2 x(k+1)\left[S_{2}(\xi)-S_{1}(\xi) D(\xi)\right] x(k-h(k)) \\
& +x^{T}(k-h(k))\left[-S_{2}(\xi) D(\xi)-D^{T}(\xi) S_{2}^{T}(\xi)\right] \\
& \times x(k-h(k)) \\
& \left.+\omega^{T}(k)\left[-2 \sigma^{T} \sigma\right] \omega(k)\right] .
\end{aligned}
$$

Applying assumption (4), the following estimations holds:

$$
\begin{gathered}
-\sigma^{T}(x(k), x(k-h(k)), k) \sigma_{i}(x(k), x(k-h(k)), k) \\
\leq \rho_{1} x^{T}(k) x(k)+\rho_{2} x^{T}(k-h(k)) x(k-h(k)) .
\end{gathered}
$$


Therefore, we have

$$
\begin{aligned}
& E\left[\Delta V_{1}(k)\right] \\
&=E\left[x ^ { T } ( k ) \left[-P(\xi)-S_{1}(\xi) A(\xi)-A(\xi)^{T} S_{1}^{T}(\xi)\right.\right. \\
&\left.+2 \rho_{1} I\right] x(k) \\
&+2 x^{T}(k)\left[S_{1}(\xi)-S_{1}(\xi) A(\xi)\right] x(k+1) \\
&+2 x^{T}(k)\left[-S_{1}(\xi) D(\xi)-S_{2}(\xi) A(\xi)\right] \\
& \times x(k-h(k)) \\
&+x(k+1)\left[P(\xi)+S_{1}(\xi)+S_{1}^{T}(\xi)\right] \\
& \times x(k+1) \\
&+2 x(k+1)\left[S_{2}(\xi)-S_{1}(\xi) D(\xi)\right] \\
& \times x(k-h(k)) \\
&+x^{T}(k-h(k))\left[-S_{2}(\xi) D(\xi)-D^{T}(\xi) S_{2}^{T}(\xi)\right. \\
&\left.\left.+2 \rho_{2} I\right] x(k-h(k))\right] .
\end{aligned}
$$

The expectation of the difference of $V_{2}(k)$ is given by

$$
\begin{aligned}
E[ & \left.\Delta V_{2}(k)\right] \\
=E[ & \sum_{i=k+1-h(k+1)}^{k} x^{T}(i) Q(\xi) x(i) \\
& \left.-\sum_{i=k-h(k)}^{k-1} x^{T}(i) Q(\xi) x(i)\right] \\
=E[ & \sum_{i=k+1-h(k+1)}^{k-h_{1}} x^{T}(i) Q(\xi) x(i) \\
& +x^{T}(k) Q(\xi) x(k) \\
& -x^{x}(k-h(k)) Q(\xi) x(k-h(k)) \\
& +\sum_{i=k+1-h_{1}}^{k-1} x^{T}(i) Q(\xi) x(i) \\
& \left.\quad-\sum_{i=k+1-h(k)}^{k-1} x^{T}(i) Q(\xi) x(i)\right] .
\end{aligned}
$$

Since $h(k) \geq h_{1}$, we have

$$
\sum_{i=k+1-h_{1}}^{k-1} x^{T}(i) Q(\xi) x(i)-\sum_{i=k+1-h(k)}^{k-1} x^{T}(i) Q(\xi) x(i) \leq 0,
$$

and, hence, from (22), we have

$$
\begin{aligned}
& E\left[\Delta V_{2}(k)\right] \\
& \quad \leq E\left[\sum_{i=k+1-h(k+1)}^{k-h_{1}} x^{T}(i) Q(\xi) x(i)\right. \\
& \quad+x^{T}(k) Q(\xi) x(k) \\
& \left.\quad-x^{T}(k-h(k)) Q(\xi) x(k-h(k))\right] .
\end{aligned}
$$

The difference of $V_{3}(k)$ is given by

$$
\begin{array}{r}
E\left[\Delta V_{3}(k)\right] \\
=E\left[\sum _ { j = - h _ { 2 } + 2 } ^ { - h _ { 1 } + 1 } \left[x^{T}(k) Q(\xi) x(k)\right.\right. \\
-x^{T}(k+j-1) Q(\xi) \\
\times x(k+j-1)]] \\
=E\left[\begin{array}{l}
\left(h_{2}-h_{1}\right) x^{T}(k) Q(\xi) x(k) \\
\left.-\sum_{l=k+1-h_{2}}^{k-h_{1}} x^{T}(l) Q(\xi) x(l)\right] .
\end{array}\right.
\end{array}
$$

Since

$$
\sum_{i=k=1-h(k+1)}^{k-h_{1}} x^{T}(i) Q(\xi) x(i)-\sum_{i=k+1-h_{2}}^{k-h_{1}} x^{T}(i) Q(\xi) x(i) \leq 0,
$$

we obtain from (24) and (25) that

$$
\begin{aligned}
& E\left[\Delta V_{2}(k)+\Delta V_{3}(k)\right] \\
& \leq E\left[\left(h_{2}-h_{1}+1\right) x^{T}(k) Q(\xi) x(k)\right. \\
& \left.\quad-x^{T}(k-h(k)) Q(\xi) x(k-h(k))\right] .
\end{aligned}
$$

Therefore, combining the inequalities (21), (27) gives

$$
E[\Delta V(k)] \leq E\left[\psi^{T}(k) T(\xi) \psi(k)\right],
$$

where 


$$
\begin{aligned}
\psi(k) & =[x(k) x(k+1) x(k-h(k))]^{T}, \\
M(\xi) & =\left(\begin{array}{ccc}
M(\xi) & S_{1}(\xi)-S_{1}(\xi) A(\xi) & -S_{1}(\xi) D(\xi)-S_{2}(\xi) A(\xi) \\
S_{1}^{T}(\xi)-A^{T}(\xi) S_{1}^{T}(\xi) & P(\xi)+S_{1}(\xi)+S_{1}^{T}(\xi) & S_{2}(\xi)-S_{1}(\xi) D(\xi) \\
-D^{T}(\xi) S_{1}^{T}(\xi)-A^{T}(\xi) S_{2}^{T}(\xi) & S_{2}^{T}(\xi)-D^{T}(\xi) S_{1}^{T}(\xi) & -Q(\xi)-S_{2}(\xi) D(\xi)-D^{T}(\xi) S_{2}^{T}(\xi)+2 \rho_{2} I
\end{array}\right), \\
M(\xi) & =\left(h_{2}-h_{1}+1\right) Q(\xi)-P(\xi)-S_{1}(\xi) A(\xi)-A(\xi)^{T} S_{1}(\xi)^{T}+2 \rho_{1} I .
\end{aligned}
$$

Let us denote that

$$
\begin{array}{cc}
M_{i j}:=\left(h_{2}-h_{1}+1\right) Q_{i}-P_{i}-S_{1 i} A_{j}-A_{j}^{T} S_{1 i}^{T}+2 \rho_{1} I, \\
\left(S_{1} A\right)_{i j}:=S_{1 j} A_{i}+S_{1 i} A_{j}, \quad\left(S_{2} A\right)_{i j}:=S_{2 j} A_{i}+S_{2 i} A_{j}, \\
\left(S_{1} D\right)_{i j}:=S_{1 j} D_{i}+S_{1 i} D_{j}, \quad\left(S_{2} D\right)_{i j}:=S_{2 j} D_{i}+S_{2 i} D_{j},
\end{array}
$$

$$
\begin{array}{cc}
P_{i j}=P_{i}+P_{j}, & Q_{i j}=Q_{i}+Q_{j}, \\
S_{1 i j}=S_{1 i}+S_{1 j}, & S_{2 i j}=S_{2 i}+S_{2 j} .
\end{array}
$$

From the convex combination of the expression of $P(\xi), Q(\xi)$, $S_{1}(\xi), S_{2}(\xi), A(\xi), D(\xi)$, we have

$$
\begin{aligned}
T(\xi)= & \sum_{i=1}^{p} \xi_{i}^{2}\left(\begin{array}{ccc}
M_{i i} & S_{1 i}-S_{1 i} A_{i} & -S_{1 i} D_{i}-S_{2 i} A_{i} \\
S_{1 i}^{T}-A_{i}^{T} S_{1 i}^{T} & P_{i}+S_{1 i}+S_{1 i}^{T} & S_{2 i}-S_{1 i} D_{i} \\
-D_{i}^{T} S_{1 i}^{T}-A_{i}^{T} S_{2 i}^{T} & S_{2 i}^{T}-D_{i}^{T} S_{1 i}^{T} & -Q_{i}-S_{2 i} D_{i}-D_{i}^{T} S_{2 i}^{T}+2 \rho_{2} I
\end{array}\right) \\
& +\sum_{i=1}^{p-1} \sum_{j=i+1}^{p} \xi_{i} \xi_{j}\left(\begin{array}{ccc}
M_{i j}+M_{j i} & S_{1 i j}-\left(S_{1} A\right)_{i j} & -\left(S_{1} D\right)_{i j}-\left(S_{2} A\right)_{i j} \\
S_{1 i j}^{T}-\left(A^{T} S_{1}^{T}\right)_{i j} & P_{i j}+S_{1 i j}+S_{1 i j}^{T} & S_{2 i j}-\left(S_{1} D\right)_{i j} \\
-\left(D^{T} S_{1}^{T}\right)_{i j}-\left(A^{T} S_{2}^{T}\right)_{i j} & S_{2 i j}^{T}-\left(D^{T} S_{1}^{T}\right)_{i j} & -Q_{i j}-\left(S_{2} D\right)_{i j}-\left(D^{T} S_{2}^{T}\right)_{i j}+2 \rho_{2} I
\end{array}\right) \\
= & \sum_{i=1}^{p} \xi_{i}^{2} \mathscr{M}_{i i}\left(P, Q, S_{1}, S_{2}\right)+\sum_{i=1}^{p-1} \sum_{j=i+1}^{p} \xi_{i} \xi_{j}\left[\mathscr{M}_{i j}\left(P, Q, S_{1}, S_{2}\right)+\mathscr{M}_{j i}\left(P, Q, S_{1}, S_{2}\right)\right] .
\end{aligned}
$$

Then, the conditions (i) and (ii) give

$$
T(\xi)<-\sum_{i=1}^{p} \xi_{i}^{2} \mathcal{S}+\frac{2}{p-1} \sum_{i=1}^{p-1} \sum_{j=i+1}^{p} \xi_{i} \xi_{j} \mathcal{S} \leq 0,
$$

because of Proposition 3 as

$$
(p-1) \sum_{i=1}^{p} \xi_{i}^{2}-2 \sum_{i=1}^{p-1} \sum_{j=i+1}^{p} \xi_{i} \xi_{j}=\sum_{i=1}^{p-1} \sum_{j=i+1}^{p}\left(\xi_{i}-\xi_{j}\right)^{2} \geq 0,
$$

and, hence, we finally obtain from (28) that

$$
E[\Delta V(k)] \leq E\left[\psi^{T}(k) T(\xi) \psi(k)\right]<0, \quad \forall k=0,1,2, \ldots,
$$

which together with (12) and Definition 2 implies that the system (1) is robustly stable in the mean square. This completes the proof of the theorem.

Remark 5. The stability conditions of Theorem 4 are more appropriate for most of real systems since it is usually impossible in practice to know exactly the delay but lower and upper bounds are always possible.
3.2. Robust Stabilization. This section deals with a stabilization problem considered in [15] for constructing a delayed feedback controller, which stabilizes the resulting closed-loop system. The robust stability condition obtained in previous section will be applied to design a time-delayed state feedback controller for the discrete-time control system described by

$$
\begin{array}{r}
x(k+1)=A(\xi) x(k)+B(\xi) u(k) \\
+\sigma(x(k), x(k-h(k)), k) \omega(k), \\
\quad k=0,1,2, \ldots,
\end{array}
$$

where $u(k) \in R^{n}$ is the control input, and the system matrices are subjected to uncertainties and belong to the polytope $\Omega$ given by

$$
\Omega=\left\{[A, B](\xi):=\sum_{i=1}^{p} \xi_{i}\left[A_{i}, B_{i}\right], \sum_{i=1}^{p} \xi_{i}=1, \xi_{i} \geq 0\right\},
$$

where $A_{i}, B_{i}, i=1,2, \ldots, p$, are given constant matrices with appropriate dimensions. As in [8], we consider a parameterdependent delayed feedback control law

$$
u(k)=F(\xi) x(k-h(k)), \quad k=-h_{2}, \ldots, 0,
$$


where $h(k)$ is the time-varying delay function satisfying $0<$ $h_{1} \leq h(k) \leq h_{2}$, and $F(\xi)$ is the controller gain to be determined. Applying the feedback controller (37) to the system (35), the closed-loop time-delay system is

$$
\begin{array}{r}
x(k+1)=A(\xi) x(k)+B(\xi) F(\xi) x(k-h(k)) \\
+\sigma(x(k), x(k-h(k)), k) \omega(k), \\
k=0,1,2, \ldots
\end{array}
$$

Definition 6. The system (35) is robustly stabilizable in the mean square if there is a delayed feedback control (37) such that the closed-loop delay system (38) is robustly stable in the mean square.

Let

$$
\begin{gathered}
\mathscr{M}_{i j}\left(P, Q, S_{1}\right)=\left(\begin{array}{ccc}
\left(h_{2}-h_{1}+1\right) Q_{i}-P_{i}-S_{1 i} A_{j}-A_{j}^{T} S_{1 i}^{T}+2 \rho_{1} I & S_{1 i}-S_{1 i} A_{j} & -P_{i}-S_{1 i} A_{j} \\
S_{1 i}^{T}-A_{j}^{T} S_{1 i}^{T} & P_{i}+S_{1 i}+S_{1 i}^{T} & S_{1 i}-P_{i} \\
-P_{i}-A_{j}^{T} S_{1 i}^{T} & S_{1 i}^{T}-P_{i} & -Q_{i}-P_{i}-P_{i}+2 \rho_{2} I
\end{array}\right), \\
\mathcal{S}=\left(\begin{array}{lll}
S & 0 & 0 \\
0 & 0 & 0 \\
0 & 0 & 0
\end{array}\right) .
\end{gathered}
$$

The following theorem can be derived from Theorem 4 .

Theorem 7. The system (35) is robustly stabilizable in the mean square by the delayed feedback control (37), where

$$
F(\xi)=B^{T}(\xi)\left[B(\xi) B^{T}(\xi)\right]^{-1} S_{1}^{T}(\xi)\left[S_{1}(\xi) S_{1}^{T}(\xi)\right]^{-1} P(\xi),
$$

if there exist symmetric matrices $P_{i}>0, Q_{i}>0, i=1,2 \ldots, p$, and constant matrices $S_{1 i}, i=1,2, \ldots, p, S \geq 0$, satisfying the following LMIs:

(i) $\mathscr{M}_{i i}\left(P, Q, S_{1}\right)+\mathcal{S}<0, i=1,2, \ldots, p$;

(ii) $\mathscr{M}_{i j}\left(P, Q, S_{1}\right)+\mathscr{M}_{j i}\left(P, Q, S_{1}\right)-(2 /(p-1)) \mathcal{S}<0, i=$ $1,2, \ldots, p-1 ; j=i+1, \ldots, p$.

Proof. Taking $S_{1 i}=S_{2 i}$ and using the feedback control (37), the closed-loop system becomes system $\left(\Sigma_{\xi}\right)$, where $D(\xi)=$ $B(\xi) F(\xi)=S_{1}^{T}(\xi)\left[S_{1}(\xi) S_{1}^{T}(\xi)\right]^{-1} P(\xi)$. Since $S_{1}(\xi) D(\xi)=P(\xi)$, the robust stability condition of the closed-loop system (38), by Theorem 4 , is immediately derived.

Remark 8. The stabilization conditions of Theorem 7 are more appropriate for most of real systems since it is usually impossible in practice to know exactly the delay but lower and upper bounds are always possible.

\section{Numerical Examples}

To illustrate the effectiveness of the previous theoretical results, we consider the following numerical examples.

Example 9 (robust stability). Consider system $\Sigma_{\xi}$ for $p=2$, where the delay function $h(k)$ is given by

$$
\begin{array}{cc}
h(k)=1+28 \sin ^{2} \frac{k \pi}{2}, & k=0,1,2, \ldots, \\
A_{1}=\left(\begin{array}{cc}
-30.5 & 1 \\
2 & -3.5
\end{array}\right), & A_{2}=\left(\begin{array}{cc}
-35.5 & 1 \\
3 & -4.5
\end{array}\right), \\
D_{1}=\left(\begin{array}{cc}
-1.5 & 0.1 \\
0.4 & -2.15
\end{array}\right), & D_{2}=\left(\begin{array}{cc}
-2.5 & 0.2 \\
0.3 & -1.85
\end{array}\right) .
\end{array}
$$

By using the LMI Toolbox in MATLAB, the LMIs (i) and (ii) of Theorem 4 are feasible with $h_{1}=1, h_{2}=29, \rho_{1}=0.011$, $\rho_{2}=0.015$, and we use the condition in the Theorem 4 for this example. The solutions of LMI verify as follow of the form

$$
\begin{aligned}
P_{1}=\left(\begin{array}{ll}
4.6120 & 0.2565 \\
0.2565 & 3.3703
\end{array}\right), \quad P_{2}=\left(\begin{array}{ll}
2.9556 & 0.0381 \\
0.0381 & 3.5256
\end{array}\right), \\
Q_{1}=\left(\begin{array}{ll}
0.1402 & 0.0109 \\
0.0109 & 0.0145
\end{array}\right), \quad Q_{2}=\left(\begin{array}{ll}
0.3550 & 0.0101 \\
0.0101 & 0.2101
\end{array}\right), \\
S_{11}=\left(\begin{array}{cc}
-0.0596 & -0.0430 \\
0.0031 & 0.0453
\end{array}\right) \\
S_{12}=\left(\begin{array}{cc}
-0.0197 & -0.0095 \\
0.0045 & 0.0375
\end{array}\right) \\
S_{21}=\left(\begin{array}{cc}
0.0006 & 0.0250 \\
-0.0029 & -0.1615
\end{array}\right) \\
S_{22}=\left(\begin{array}{cc}
-0.0002 & 0.0133 \\
-0.0030 & -0.1228
\end{array}\right) \\
S=\left(\begin{array}{cc}
2.0759 & 0.0459 \\
0.0459 & 1.3271
\end{array}\right) .
\end{aligned}
$$

Therefore, the system is robustly stable.

Example 10 (robust stabilization). Consider system (35) for $p=2$, where the delay function $h(k)$ is given by

$$
\begin{array}{cc}
h(k)=1+34 \sin ^{2} \frac{k \pi}{2}, & k=0,1,2, \ldots, \\
A_{1}=\left(\begin{array}{cc}
-30.5 & 1 \\
2 & -3.5
\end{array}\right), & A_{2}=\left(\begin{array}{cc}
-35.5 & 1 \\
3 & -4.5
\end{array}\right), \\
B_{1}=\left(\begin{array}{cc}
-1.5 & 0.1 \\
0.4 & -2.15
\end{array}\right), & B_{2}=\left(\begin{array}{cc}
-2.5 & 0.2 \\
0.3 & -1.85
\end{array}\right) .
\end{array}
$$


By using the LMI Toolbox in MATLAB, the LMIs (i) and (ii) of Theorem 7 are feasible with $h_{1}=1, h_{2}=35, \rho_{1}=0.011$, $\rho_{2}=0.015$, and we use the condition in the Theorem 7 for this example. The solutions of LMI verify as follow of the form

$$
\begin{gathered}
P_{1}=\left(\begin{array}{cc}
1.3886 & -0.0760 \\
-0.0760 & 1.3559
\end{array}\right), \quad P_{2}=\left(\begin{array}{ll}
1.6286 & 0.0649 \\
0.0649 & 1.5243
\end{array}\right), \\
Q_{1}=\left(\begin{array}{cc}
0.0097 & -0.0048 \\
-0.0048 & 0.0057
\end{array}\right), \\
Q_{2}=\left(\begin{array}{cc}
0.0728 & -0.0159 \\
-0.0159 & 0.0621
\end{array}\right),
\end{gathered}
$$

$$
\begin{gathered}
S_{11}=\left(\begin{array}{cc}
-0.0274 & 0.0827 \\
-0.0133 & -0.2222
\end{array}\right), \\
S_{12}=\left(\begin{array}{cc}
-0.0209 & 0.0619 \\
-0.0226 & -0.1942
\end{array}\right), \\
S=\left(\begin{array}{cc}
0.5954 & -0.0672 \\
-0.0672 & 0.5469
\end{array}\right) .
\end{gathered}
$$

Therefore, the system is robustly stabilizable with the feedback control

$$
\begin{aligned}
u(k)= & B^{T}(\xi)\left[B(\xi) B^{T}(\xi)\right]^{-1} S_{1}^{T}(\xi)\left[S_{1}(\xi) S_{1}^{T}(\xi)\right]^{-1} P(\xi) x(k-h(k)) \\
= & \left(\xi_{1} B_{1}+\xi_{2} B_{2}\right)^{T}\left[\left(\xi_{1} B_{1}+\xi_{2} B_{2}\right)\left(\xi_{1} B_{1}+\xi_{2} B_{2}\right)^{T}\right]^{-1} \\
& \times\left(\xi_{1} S_{11}+\xi_{2} S_{12}\right)^{T}\left[\left(\xi_{1} S_{11}+\xi_{2} S_{12}\right)\left(\xi_{1} S_{11}+\xi_{2} S_{12}\right)^{T}\right]^{-1}\left(\xi_{1} P_{1}+\xi_{2} P_{2}\right)(\xi) x(k-h(k)) \\
= & \left(\begin{array}{ccc}
-1.5 \xi_{1}-2.5 \xi_{2} & 0.4 \xi_{1}+0.3 \xi_{2} \\
0.1 \xi_{1}+0.2 \xi_{2} & -2.15 \xi_{1}-1.85 \xi_{2}
\end{array}\right) \times\left(\begin{array}{cc}
-1.5 \xi_{1}-2.5 \xi_{2} & 0.4 \xi_{1}+0.3 \xi_{2} \\
0.1 \xi_{1}+0.2 \xi_{2} & -2.15 \xi_{1}-1.85 \xi_{2}
\end{array}\right)^{-1} \\
& \times\left(\begin{array}{ccc}
-1.5 \xi_{1}-2.5 \xi_{2} & 0.1 \xi_{1}+0.2 \xi_{2} \\
0.4 \xi_{1}+0.3 \xi_{2} & -2.15 \xi_{1}-1.85 \xi_{2}
\end{array}\right)^{-1} \times\left(\begin{array}{cc}
-0.0274 \xi_{1}-0.0209 \xi_{2} & -0.0133 \xi_{1}-0.0226 \xi_{2} \\
0.0827 \xi_{1}+0.0619 \xi_{2} & -0.2222 \xi_{1}-0.1942 \xi_{2}
\end{array}\right) \\
& \times\left(\begin{array}{ccc}
-0.0274 \xi_{1}-0.0209 \xi_{2} & -0.0133 \xi_{1}-0.0226 \xi_{2} \\
0.0827 \xi_{1}+0.0619 \xi_{2} & -0.2222 \xi_{1}-0.1942 \xi_{2}
\end{array}\right)^{-1} \times\left(\begin{array}{ll}
-0.0274 \xi_{1}-0.0209 \xi_{2} & 0.0827 \xi_{1}+0.0619 \xi_{2} \\
-0.0133 \xi_{1}-0.0226 \xi_{2} & -0.2222 \xi_{1}-0.1942 \xi_{2}
\end{array}\right) \\
& \times\left(\begin{array}{cc}
1.3886 \xi_{1}+1.6286 \xi_{2} & -0.0760 \xi_{1}+0.0649 \xi_{2} \\
-0.0760 \xi_{1}+0.0649 \xi_{2} & 1.3559 \xi_{1}+1.5243 \xi_{2}
\end{array}\right) \\
= & \left(\begin{array}{lll}
-2.0829 \xi_{1}^{2}-5.9144 \xi_{1} \xi_{2}-4.0715 \xi_{2}^{2} & -0.0304 \xi_{1}^{2}+0.0260 \xi_{1} \xi_{2}+0.0195 \xi_{2}^{2} \\
-0.0076 \xi_{1}^{2}-0.0087 \xi_{1} \xi_{2}+0.0128 \xi_{2}^{2} & -2.9152 \xi_{1}^{2}-5.7856 \xi_{1} \xi_{2}-2.8200 \xi_{2}^{2}
\end{array}\right) x(k-h(k)) .
\end{aligned}
$$

Therefore, the feedback delayed controller is

$$
\begin{aligned}
u_{1}(k)= & {\left[-2.0829 \xi_{1}^{2}-5.9144 \xi_{1} \xi_{2}-4.0715 \xi_{2}^{2}\right] } \\
& \times x_{1}(k-h(k)) \\
& +\left[-0.0304 \xi_{1}^{2}+0.0260 \xi_{1} \xi_{2}+0.0195 \xi_{2}^{2}\right] \\
& \times x_{2}(k-h(k)), \\
u_{2}(k)= & {\left[-0.0076 \xi_{1}^{2}-0.0087 \xi_{1} \xi_{2}+0.0128 \xi_{2}^{2}\right] } \\
& \times x_{1}(k-h(k)) \\
& +\left[-2.9152 \xi_{1}^{2}-5.7856 \xi_{1} \xi_{2}-2.8200 \xi_{2}^{2}\right] \\
& \times x_{2}(k-h(k)) .
\end{aligned}
$$

\section{Conclusion}

In this paper, new delay-dependent mean square robust stability conditions for linear polytopic delay-difference stochastic equations with interval time-varying delays have been presented in terms of LMIs. An application to mean square robust stabilization of discrete stochastic control systems with time-delayed feedback controllers has been studied. Numerical examples have been given to demonstrate the effectiveness of the proposed conditions.

\section{Acknowledgments}

This work was supported by the Office of Agricultural Research and Extension Maejo University, the Thailand Research Fund Grant, the Higher Education Commission, and Faculty of Science, Maejo University, Thailand. The first author is supported by the Center of Excellence in Mathematics, Thailand, and Commission for Higher Education, Thailand. The authors thank anonymous reviewers for valuable comments and suggestions, which allowed them to improve the paper.

\section{References}

[1] R. P. Agarwal, Difference Equations and Inequalities, vol. 228, Marcel Dekker Inc., New York, NY, USA, 2nd edition, 2000.

[2] W. H. Chen, Z. H. Guan, and X. Lu, "Delay-dependent guaranteed cost control for uncertain discrete-time systems with 
delay,' IEE Proceedings: Control Theory and Applications, vol. 150, no. 4, pp. 412-416, 2003.

[3] S. Elaydi and I. Győri, "Asymptotic theory for delay difference equations," Journal of Difference Equations and Applications, vol. 1, no. 2, pp. 99-116, 1995.

[4] V. Kolmanovskii and A. Myshkis, Applied Theory of Functional Differential Equations, Springer, Berlin, Germany, 1992.

[5] O. M. Kwon and J. H. Park, "Exponential stability of uncertain dynamic systems including state delay," Applied Mathematics Letters, vol. 19, no. 9, pp. 901-907, 2006.

[6] W. J. Mao and J. Chu, "D-stability and D-stabilization of linear discrete time-delay systems with polytopic uncertainties," Automatica, vol. 45, no. 3, pp. 842-846, 2009.

[7] P. T. Nam, H. M. Hien, and V. N. Phat, "Asymptotic stability of linear state-delayed neutral systems with polytope type uncertainties," Dynamic Systems and Applications, vol. 19, no. 1, pp. 63-72, 2010.

[8] V. N. Phat and J. Y. Park, "On the Gronwall inequality and asymptotic stability of nonlinear discrete systems with multiple delays," Dynamic Systems and Applications, vol. 10, no. 4, pp. 577-588, 2001.

[9] T. L. Hsien and C. H. Lee, "Exponential stability of discrete time uncertain systems with time-varying delay," Journal of the Franklin Institute, vol. 332, no. 4, pp. 479-489, 1995.

[10] D. H. Ji, J. H. Park, W. J. Yoo, and S. C. Won, "Robust memory state feedback model predictive control for discretetime uncertain state delayed systems," Applied Mathematics and Computation, vol. 215, no. 6, pp. 2035-2044, 2009.

[11] G. Rajchakit, T. Rojsiraphisal, and M. Rajchakit, "Robust stability and stabilization of uncertain switched discrete-time systems," Advances in Difference Equations, vol. 2012, article 134, 2012.

[12] E. K. Boukas, "State feedback stabilization of nonlinear discretetime systems with time-varying time delay," Nonlinear Analysis. Theory, Methods \& Applications., vol. 66, no. 6, pp. 1341-1350, 2007.

[13] H. Gao and T. Chen, "New results on stability of discrete-time systems with time-varying state delay," IEEE Transactions on Automatic Control, vol. 52, no. 2, pp. 328-334, 2007.

[14] X. Jiang, Q. L. Han, and X. Yu, "Stability criteria for linear discrete-time systems with interval-like time-varying delay," in Proceedings of the American Control Conference (ACC '05), pp. 2817-2822, June 2005.

[15] B. Zhang, S. Xu, and Y. Zou, "Improved stability criterion and its applications in delayed controller design for discrete-time systems," Automatica, vol. 44, no. 11, pp. 2963-2967, 2008.

[16] M. Yu, L. Wang, and T. Chu, "Robust stabilization of discretetime systems with time-varying delays," in Proceedings of the American Control Conference (ACC '05), pp. 3435-3440, Portland, Ore, USA, June 2005.

[17] K. Ratchagit and V. N. Phat, "Stability criterion for discretetime systems," Journal of Inequalities and Applications, vol. 2010, Article ID 201459, 6 pages, 2010.

[18] G. Zhai, B. Hu, K. Yasuda, and A. N. Michel, "Qualitative analysis of discrete-time switched systems," in Proceedings of the American Control Conference (ACC '02), pp. 1880-1885, May 2002.

[19] M. Rajchakit, P. Niamsup, and G. Rajchakit, "A switching rule for exponential stability of switched recurrent neural networks with interval time-varying delay," Advances in Difference Equations, vol. 2013, article 44, 2013.
[20] K. Ratchagit, "A switching rule for the asymptotic stability of discrete-time systems with convex polytopic uncertainties," Asian-European Journal of Mathematics, vol. 5, no. 2, Article ID 1250025, 12 pages, 2012.

[21] M. Rajchakit and G. Rajchakit, "Mean square exponential stability of stochastic switched system with interval timevarying delays," Abstract and Applied Analysis, vol. 2012, Article ID 623014, 12 pages, 2012.

[22] W. A. Zhang and L. Yu, "Stability analysis for discrete-time switched time-delay systems," Automatica, vol. 45, no. 10, pp. 2265-2271, 2009.

[23] V. N. Phat and K. Ratchagit, "Stability and stabilization of switched linear discrete-time systems with interval time-varying delay," Nonlinear Analysis: Hybrid Systems, vol. 5, no. 4, pp. 605-612, 2011.

[24] M. O. Abou Al-Ola, K. Fujimoto, and T. Yoshinaga, "Common Lyapunov function based on Kullback-Leibler divergence for a switched nonlinear system," Mathematical Problems in Engineering, vol. 2011, Article ID 723509, 12 pages, 2011.

[25] Y. He, M. Wu, J. H. She, and G. P. Liu, "Parameter-dependent Lyapunov functional for stability of time-delay systems with polytopic-type uncertainties," IEEE Transactions on Automatic Control, vol. 49, no. 5, pp. 828-832, 2004.

[26] D. Henrion, D. Arzelier, D. Peaucelle, and M. Šebek, "LMI condition for robust stability of polynomial matrix polytopes," Automatica, vol. 37, no. 3, pp. 461-468, 2001.

[27] D. F. Coutinho, M. Fu, and A. Trofino, "Robust analysis and control for a class of uncertain nonlinear discrete-time systems," Systems and Control Letters, vol. 53, no. 5, pp. 377-393, 2004.

[28] S. W. Kau, Y. Liu, L. Hang, C. H. Lee, C. H. Fang, and L. Lee, "A new LMI condition for robust stability of discrete-time uncertain systems," Applied Mathematics and Computation, vol. 215, pp. 2035-2044, 2009.

[29] M. Rajchakit and G. Rajchakit, "Mean square robust stability of stochastic switched discrete-time systems with convex polytopic uncertainties," Journal of Inequalities and Applications, vol. 2012, article 135, 2012.

[30] B. Du and X. Zhang, "Delay-dependent stability analysis and synthesis for uncertain impulsive switched system with mixed delays," Discrete Dynamics in Nature and Society, vol. 2011, Article ID 381571, 9 pages, 2011.

[31] M. De la Sen and A. Ibeas, "Stability results of a class of hybrid systems under switched continuous-time and discretetime control," Discrete Dynamics in Nature and Society, vol. 2009, Article ID 315713, 28 pages, 2009.

[32] M. de la Sen and A. Ibeas, "Stability results for switched linear systems with constant discrete delays," Mathematical Problems in Engineering, vol. 2008, Article ID 543145, 28 pages, 2008.

[33] M. Rajchakit and G. Rajchakit, "LMI approach to robust stability and stabilization of nonlinear uncertain discrete-time systems with convex polytopic uncertainties," Advances in Difference Equations, vol. 2012, 2012, article 106.

[34] J. Li, W. Wu, J. Yuan, Q. Tan, and X. Yin, "Delay-dependent stability criterion of arbitrary switched linear systems with time-varying delay," Discrete Dynamics in Nature and Society, vol. 2010, Article ID 347129, 16 pages, 2010.

[35] L. Zhang, H. Li, and Y. Chen, "Robust stability analysis and synthesis for switched discrete-time systems with time delay," Discrete Dynamics in Nature and Society, vol. 2010, Article ID 408105, 19 pages, 2010. 
[36] M. De La Sen, "On the characterization of hankel and toeplitz operators describing switched linear dynamic systems with point delays," Abstract and Applied Analysis, vol. 2009, Article ID 670314, 34 pages, 2009. 


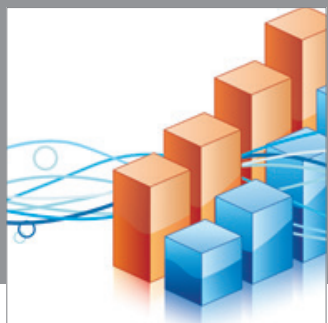

Advances in

Operations Research

mansans

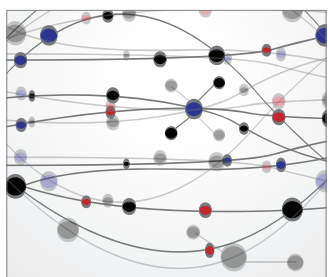

The Scientific World Journal
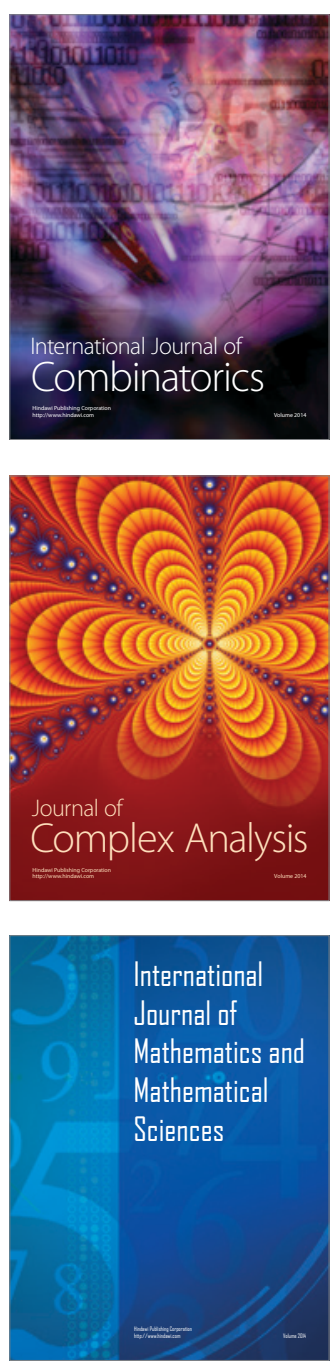
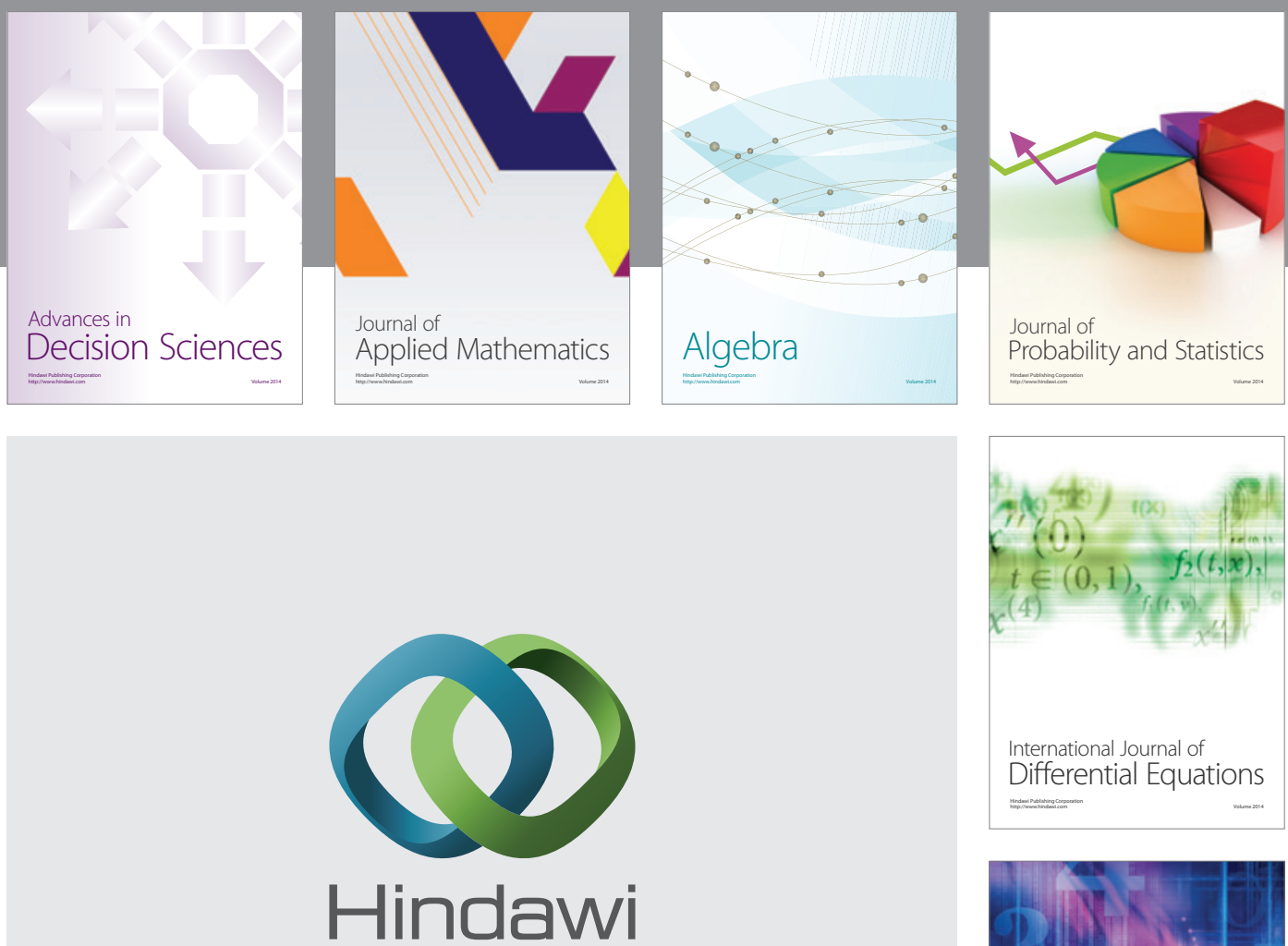

Submit your manuscripts at http://www.hindawi.com
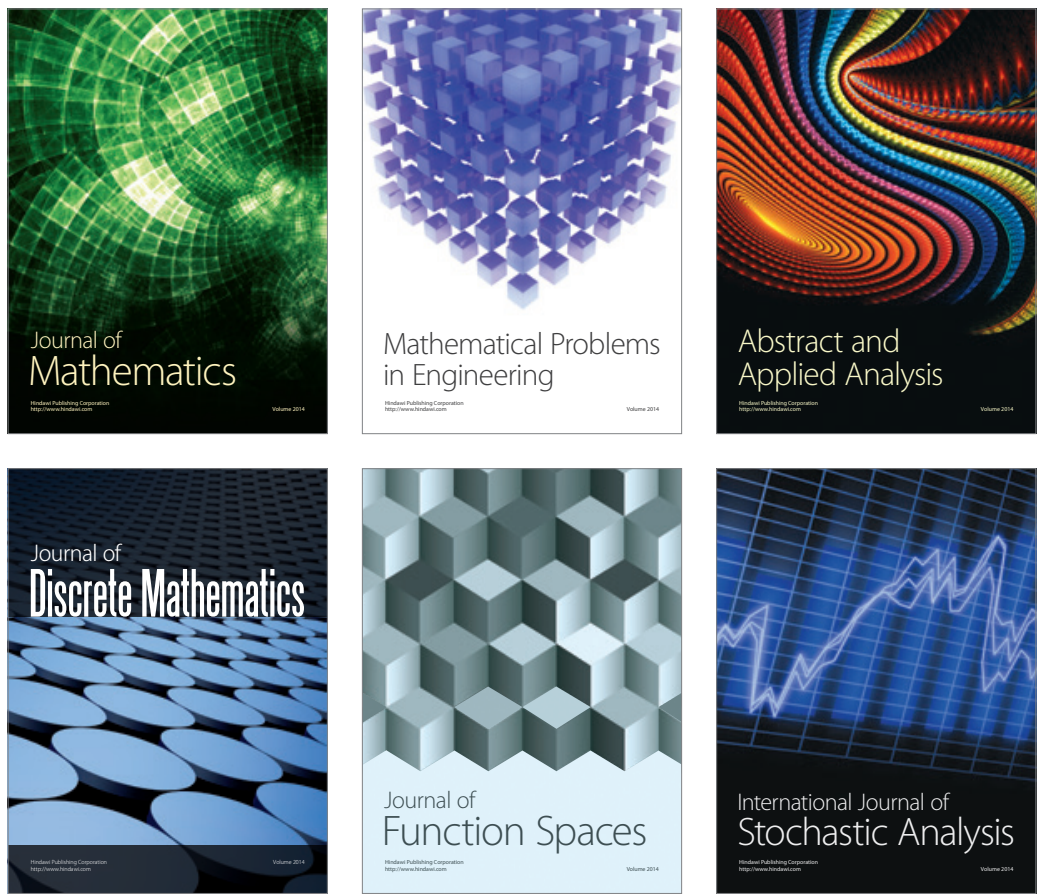

Journal of

Function Spaces

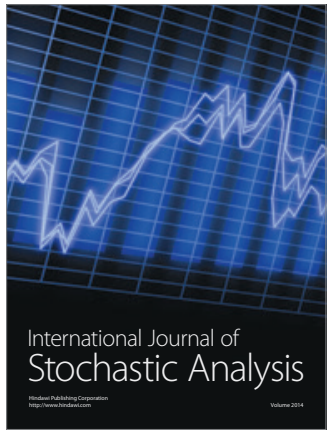

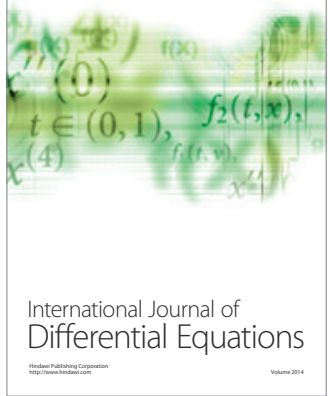
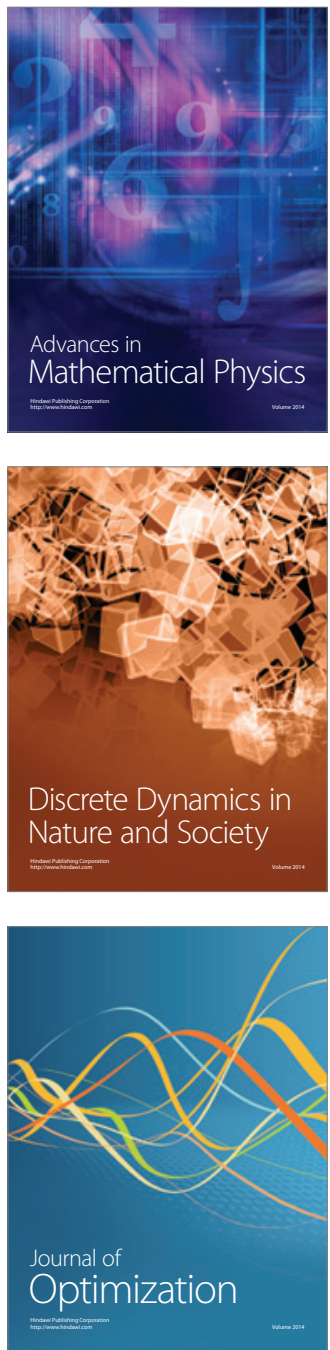